\title{
Qualitative and quantitative analyses of influenza virus receptors in trachea and lung tissues of humans, mice, chickens and ducks
}

\author{
WANG BaoLin ${ }^{1}$, LIU Bo $^{1}$, CHEN Ling ${ }^{1 *}$, ZHANG JianYong ${ }^{1}$, \\ HE HongXuan ${ }^{2} \&$ ZHANG Hong ${ }^{1,2,3^{*}}$ \\ ${ }^{1}$ Laboratory of Respiratory Medicine, Department of Respiratory Medicine, Affiliated Hospital of Zunyi Medical College, \\ Zunyi 563003, China; \\ ${ }^{2}$ National Research Center for Wildlife-borne Diseases, Institute of Zoology, Chinese Academy of Sciences, Beijing 100101, China; \\ ${ }^{3}$ Z-BioMed, Inc., Rockville, MD 20855, USA
}

Received February 17, 2012 ; accepted April 11, 2012

\begin{abstract}
To accurately determine the expression and distribution patterns of two influenza virus receptors (SA 2 2,3-gal and SA $\alpha 2,6$-gal) in trachea and lung tissues of humans, mice, chickens and ducks, we analyzed lectin immunofluorescence stainings of various tissue sections qualitatively and quantitatively. Results from the qualitative analysis showed that both influenza virus receptors were expressed in lung tissues of humans, mice, chickens and ducks as well as trachea tissues of mice and ducks. However, SA 2,6-gal receptor was expressed only in the human trachea tissue and SA 2,3-gal receptor was expressed only in the chicken trachea tissue. Results from the quantitative analysis demonstrated that both receptors were expressed in trachea tissues of human and mouse, as well as in lung tissues of humans, chickens and ducks. Meanwhile, our results also showed that the expression and distribution of influenza virus receptors in the same tissue were not always uniform, indicating that their distribution and expression in various tissues are not simply the distinction between the presence or absence of receptors, but rather the difference in the amount of expressed receptors.
\end{abstract}

influenza virus, sialic acid receptors, trachea, lung, lectin, immunofluorescence, quantitative analysis, qualitative analysis

Citation: Wang B L, Liu B, Chen L, et al. Qualitative and quantitative analyses of influenza virus receptors in trachea and lung tissues of humans, mice, chickens and ducks. Sci China Life Sci, 2012, 55: 612-617, doi: 10.1007/s11427-012-4341-8

The 2009 pandemic H1N1 influenza and outbreaks of the highly pathogenic H5N1 avian influenza in Hong Kong in 1997 severely threatened human health. The ability of influenza viruses to infect host cells depends mainly on interactions between the major viral surface protein, hemagglutinin, and virus receptors on host cells; therefore, the distribution and expression status of influenza virus receptors in trachea and lung tissues of different hosts may be one of the key factors in determining whether influenza viruses are capable of invading host cells and causing disease. Sialic acid alpha 2,3-galactose (SA 2 2,3-gal) and sialic acid alpha

*Corresponding author (email: lingjuncd@yahoo.com; hzhang@zbiomed.com) 2,6-galactose (SA 2,6-gal) are two well-known types of influenza virus receptors. It is generally accepted that avian influenza viruses preferentially bind to the avian influenza receptor (SA 2,3 -gal), while human influenza viruses preferentially bind to the human influenza receptor (SA 2,6gal). However, reports on the distribution of these two types of receptors in different host tissues and the tissue tropism of various influenza viruses have been contradictory [1-4]. One plausible explanation is that the conclusions of some studies were based on qualitative analysis of a limited number of stained cells in immunohistochemistry experiments using lectins or influenza viruses [4]. Therefore, it is necessary to use a quantitative method to precisely deter- 
mine the distribution and expression characteristics of these two types of influenza virus receptors in trachea and lung tissues of humans, mice, chickens and ducks, which may help us to better understand the mechanism of how different influenza viruses could infect these host tissues. Currently, plant lectins have been widely used for detecting specific sialic acid linkages. Maackia amurensis lectin (MAA) can specifically recognize $\alpha 2,3$-linked sialic acids [5], as a marker for the avian influenza virus receptor, while Sambucus nigra agglutinin (SNA) can specifically recognize $\alpha 2,6$-linked sialic acids [6], as a marker for the human influenza virus receptor. In this study, we utilized lectin immunofluorescence staining to systematically analyze the distribution and expression status of two types of sialic acid receptors in trachea and lung tissues of humans, mice, chickens and ducks to help us better understand the distribution characteristics and expression status of the two types of influenza virus sialic acid receptors in trachea and lung tissues of various hosts.

\section{Materials and methods}

\subsection{Materials and reagents}

Paraffin-embedded human trachea and lung tissue sections were obtained from autopsy samples from 2009 (Department of Pathology, Affiliated Hospital of Zunyi Medical College, Guizhou, China). Sixty mice (6-8 week old male BALB/c mice) were purchased from Shanghai Slrc Laboratory Animal Center. Twelve chickens and twelve ducks were purchased from a Zunyi poultry market, and 2 SPF grade chickens were purchased from Beijing Merial Vital Laboratory Animal Center. Two additional ducks were purchased from a poultry farm located in suburban Beijing. Biotinylated SNA (Sambucus nigra agglutinin), MAA-I (Maackia amurensis agglutinin I), MAA-II (Maackia amurensis agglutinin II), and Fluorescein Avidin DCS were purchased from Vector Laboratories (Burlingame, CA). DAPI (4',6-diamidino-2-phenylindole) was purchased from Beijing Solarbio Inc. (China), polylysine was purchased from Sigma (St. Louis, MO) and slides were purchased from Jiangsu Haimen Lab Suppliers Inc. (China).

\subsection{Treatment of tissue samples and immunofluores- cence staining}

Mice were anesthetized by intraperitoneal injection of $10 \%$ chloral hydrate and sacrificed through bleeding from abdominal aorta. Trachea and the right middle lobe lung tissues (about $0.5 \mathrm{~cm} \times 0.5 \mathrm{~cm} \times 0.2 \mathrm{~cm}$ ) were obtained and rinsed twice with saline solution, and then put immediately into $10 \mathrm{~mL}$ of $4 \%$ paraformaldehyde and fixed for $24 \mathrm{~h}$ at room temperature $\left(15-25^{\circ} \mathrm{C}\right)$. Chickens and ducks were sacrificed through bleeding, and trachea and the right mid- dle lobe lung tissues (about $1 \mathrm{~cm} \times 1 \mathrm{~cm} \times 0.5 \mathrm{~cm}$ ) were obtained as described above for mouse tissue. Fixed trachea and lung tissues of humans, mice, chickens and ducks were dehydrated, imbedded in paraffin and cut into $4 \mu \mathrm{m}$ sections. Standard procedures were followed for HE and immunofluorescence staining [7]. Results of HE staining were used to determine whether tissue structures were intact. For immunofluorescence staining, each tissue section was attached to slides pretreated with polylysine solution, and biotinylated lectins SNA, MAA-I and MAA-II and PBS (as a blank control) were added separately to four slides for each tissue. The following procedures were followed for immunofluorescence staining: tissue sections were deparaffinized and hydrated using xylene and gradient-alcohol, antigens were retrieved by heating samples in a microwave for $15 \mathrm{~min}$ in citric acid buffer (1.0 mol L $\left.\mathrm{m}^{-1} \mathrm{pH} 6.0\right)$, cooled to room temperature, and then $50 \mu \mathrm{L}$ of SNA $\left(20 \mu \mathrm{g} \mathrm{mL}^{-1}\right)$, MAA-I (20 $\left.\mu \mathrm{g} \mathrm{mL}^{-1}\right)$, MAA-II (40 $\left.\mu \mathrm{g} \mathrm{mL}^{-1}\right)$ and PBS were added separately to each slide and incubated for $1 \mathrm{~h}$ at room temperature. After washing three times with TPBS, $50 \mu \mathrm{L}$ of fluorescein avidin DCS $\left(5 \mu \mathrm{g} \mathrm{mL}^{-1}\right)$ was added to each slide and slides were incubated for $30 \mathrm{~min}$ at room temperature. Slides were washed again three times with TPBS and mounted after adding a drop of DAPI solution $\left(10 \mu \mathrm{g} \mathrm{mL}^{-1}\right)$ to stain for nuclei.

\subsection{Microscopic observation and quantification}

Immunofluorescence stained trachea and lung tissues were observed under a LEICA fluorescence microscope (Model: DM4000B, Germany), and images were collected using the software Leica Qwin Plus. The number of total cells and stained cells were counted for statistical analysis to get average positive rates.

\section{Results}

2.1 Distribution and expression of influenza virus sialic acid receptors in trachea tissues of humans, mice, chickens and ducks

\subsubsection{Qualitative analysis}

In the present study, we observed various immunofluorescence-stained trachea tissue sections under a fluorescence microscope and defined stained cells, which were clearly stronger than the background, as positive. Results showed that positively stained cells were mainly distributed on epithelia and mucus cells of tracheas. SNA stained cells were only observed on epithelium cells of human trachea (Figure 1A), while MAA-I, MAA-II and SNA stained cells were detected on epithelium cells of mouse (Figure 1B). MAA-I and MAA-II stained cells but not SNA stained cells were observed on epithelium cells and mucosa of chicken trachea 

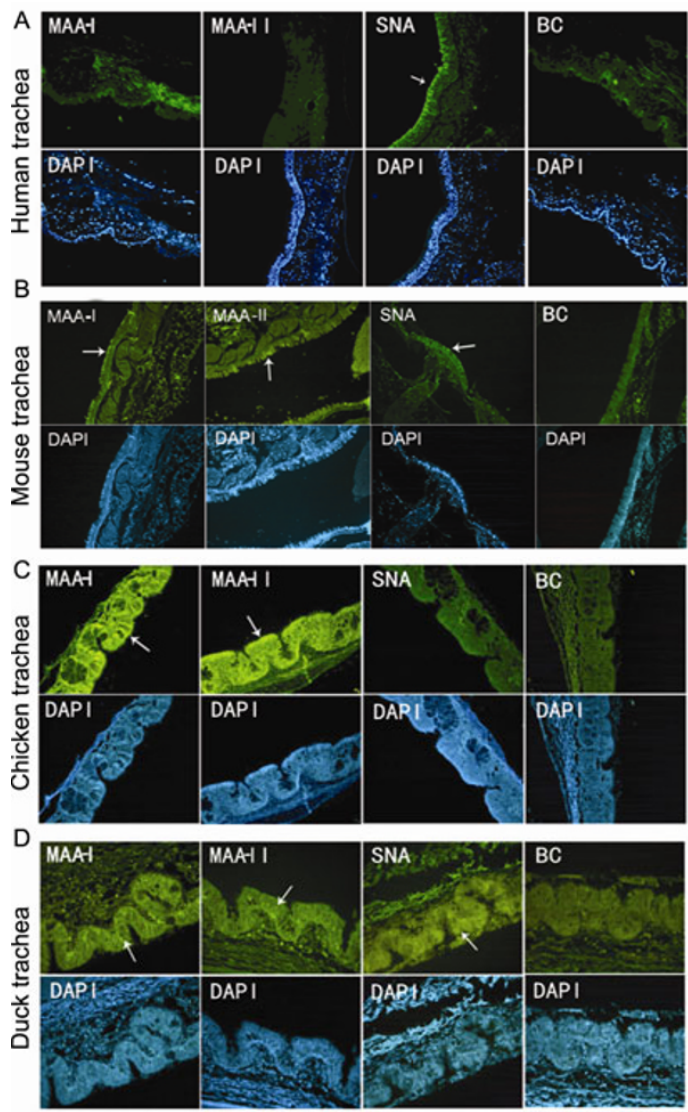

Figure 1 Distribution and expression of two types of influenza virus sialic acid receptors in trachea tissues of humans, mice, chickens and ducks. A, Human trachea. B, Mouse trachea. C, Chicken trachea. D, Duck trachea. Biotinylated MAA-I, MAA-II and SNA were used in the immunofluorescence staining of tissue sections followed by Fluorescein Avidin DCS. Arrows indicate positive staining. DAPI was used for the nuclear staining. BC, blank control. Enlargement, 200x.

(Figure 1C). Positive staining was detected on MAA-I, MAA-II and SNA stained cells in duck trachea tissues (Figure 1D). Results of the qualitative analysis demonstrated that both SA $\alpha 2,3$-gal and SA 2 2,6-gal receptors were expressed in trachea tissues of mice and ducks. However, the SA 2 2,6-gal receptor was only expressed in human trachea tissue whereas the SA $\alpha 2,3$-gal receptor was only expressed in chicken trachea tissue.

\subsubsection{Quantitative analysis}

We selected one stained slide for each trachea tissue sample, examined 5-6 fields randomly using a fluorescence micro- scope $(200 \times)$, counted the total number of trachea epithelial cells (866-3623) and the number of positively stained cells (28-281), and then calculated the average positive rate of lectin staining. It was found that the nuclei of trachea epithelium cells of chickens and ducks were faintly stained by DAPI, thus the total number of cells could not be obtained for these samples. We repeated the experiment at the National Research Center for Wildlife-borne Diseases, Institute of Zoology, Chinese Academy of Sciences and obtained the same results. In trachea tissues of humans and mice, the positive rates of lectin staining were: MAA-I, $7.8 \%$ and $5.1 \%$; MAA-II, $5.0 \%$ and $5.2 \%$; and SNA, $6.0 \%$ and $7.8 \%$, respectively (Table 2 ). False positive staining in PBS control samples was also observed in trachea epithelium cells of humans and mice at rates of $3.2 \%$ and $4.9 \%$, respectively (Table 2). Results of the quantitative analysis showed that both influenza virus sialic acid receptors were expressed in trachea tissues of humans and mice, and basically the same results were obtained when MAA-I and MAA-II were used for immunofluorescence staining.

By comparing the two methods, we found that results from the quantitative analysis were essentially consistent with those from the qualitative analysis in most cases. However, sometimes results from the two analyses were different. For example, it was observed in the qualitative analysis that there was no significantly positive staining of MAA-I and MAA-II in the human trachea tissue (Figure 1A, Table 1), meaning that avian influenza virus receptor (SA 2 2,3-gal) was not expressed on human trachea epithelium cells. Nevertheless, results from quantitative analysis showed that two types of influenza virus receptors were expressed in the human trachea tissue (Table 2), but positive rates were different (MAA-I, 7.8\%; MAA-II, 5.0\%; SNA, $6.0 \%)$.

\subsection{Distribution and expression of influenza virus sialic acid receptors in lung tissues of humans, mice, chickens and ducks}

\subsubsection{Qualitative analysis}

In the qualitative analysis of lectin immunofluorescence staining results of lung tissues, cells which were clearly more intensely stained than the background were defined as positive ones. Results from the qualitative analysis showed that cells expressing influenza virus sialic acid receptors were mainly distributed on alveolar surfaces and terminal

Table 1 Results of qualitative analysis of two influenza virus sialic acid receptors in trachea and lung tissues of humans, mice, chickens and ducks

\begin{tabular}{|c|c|c|c|c|c|c|c|c|}
\hline & \multicolumn{2}{|c|}{ Human } & \multicolumn{2}{|c|}{ Mouse } & \multicolumn{2}{|c|}{ Chicken } & \multicolumn{2}{|c|}{ Duck } \\
\hline & Trachea & Lung & Trachea & Lung & Trachea & Lung & Trachea & Lung \\
\hline MAA-I $(\alpha-2,3)$ & - & + & + & + & + & + & + & + \\
\hline MAA-II $(\alpha-2,3)$ & - & + & + & + & + & + & + & + \\
\hline $\operatorname{SNA}(\alpha-2,6)$ & + & + & + & + & - & + & + & + \\
\hline
\end{tabular}

a) + represents positive staining; - represents negative staining. 
bronchioles (Figure 2). There was positive staining of lectins MAA-I, MAA-II and SNA in lung tissues of humans, mice, chickens and ducks (Figure 2A-D). Results from the qualitative analysis indicated that two types of influenza virus receptors, SA $\alpha 2,3$-gal and SA 2 2,6-gal, were expressed in lung tissues of humans, mice, chickens and ducks (Figure 2, Table 1).

\subsubsection{Quantitative analysis}

We selected one stained slide for each lung tissue sample,

Table 2 Results of quantitative analysis of two influenza virus sialic acid receptors in trachea tissues of humans and mice

\begin{tabular}{lccc}
\hline & Human & & Mouse \\
\cline { 2 - 2 } & $\begin{array}{c}\text { Positive cells/cells } \\
\text { counted }(\%)\end{array}$ & $\begin{array}{c}\text { Positive cells/cells } \\
\text { counted }(\%)\end{array}$ \\
\hline MAA-I $(\alpha-2,3)$ & $89 / 1139(7.8)$ & & $159 / 3098(5.1)$ \\
MAA-II $(\alpha-2,3)$ & $70 / 1414(5.0)$ & & $186 / 3578(5.2)$ \\
SNA $(\alpha-2,6)$ & $79 / 1309(6.0)$ & & $281 / 3623(7.8)$ \\
PBS control & $28 / 866(3.2)$ & & $131 / 2696(4.9)$ \\
\hline
\end{tabular}
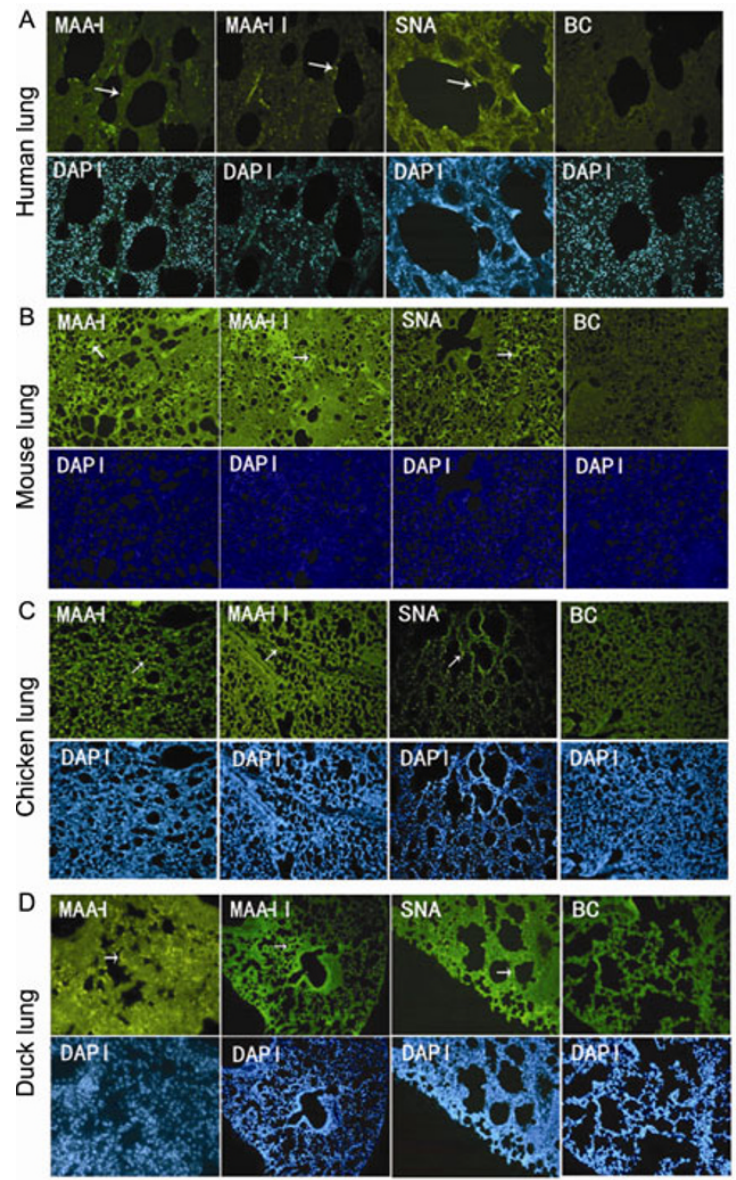

Figure 2 Distribution and expression of two influenza virus sialic acid receptors in lung tissues of humans, mice, chickens and ducks. A, Human trachea. B, Mouse trachea. C, Chicken trachea. D, Duck trachea. Biotinylated MAA-I, MAA-II and SNA were used in the immunofluorescence staining of tissue sections followed by fluorescein avidin DCS. Arrows indicate positive staining. DAPI was used for nuclear staining. BC, blank control. Objective, $200 \times$. examined 5-6 fields randomly under a microscope (200x), counted the total number of cells in the lung tissue section (3024-16174) and the number of positively stained cells (124-707), and then calculated the average positive rate of lectin staining. Results of quantitative analysis showed that the positive rates of MAA-I staining in lung tissues of humans, mice, chickens and ducks were $4.5 \%, 3.5 \%, 4.4 \%$, and $2.7 \%$; respectively. The positive rates of MAA-II staining in lung tissues of humans, mice, chickens and ducks were $4.6 \%, 3.7 \%, 3.8 \%$, and $2.6 \%$, respectively, while the positive rates of SNA staining these tissues were $4.2 \%$, $4.1 \%, 5.2 \%$, and $2.6 \%$, respectively. During the calculation process, we also found some false positive cells in the negative control, and the false positive rates for lung tissues of humans, mice, chickens and ducks were $3.2 \%, 4.1 \%, 2.2 \%$ and $2.2 \%$, respectively (Table 3 ).

Results from the quantitative analysis indicated that two types of influenza virus sialic acid receptors were expressed in lung tissues of humans, mice, chickens and ducks. Similar results were obtained when biotinylated lectins MAA-I and MAA-II were used for immunofluorescence staining. The background for the blank control in the immunofluorescence staining of mouse lung tissue was high $(4.1 \%)$, which prevented us from quantitatively analyzing the distribution and expression of influenza virus receptors in this tissue. One possible reason for this high background may be due to relatively more endogenous biotin in the normal lung tissues of mice compared to lung tissues of humans, chickens and ducks.

\section{Discussion}

Specific interaction between influenza viruses with different host cell surface receptors is considered to be an important factor in determining whether influenza viruses can invade host cells and spread across host species barriers. It was believed that receptors for avian influenza viruses were not present in tissues of the human upper respiratory tract, and consequently, avian influenza viruses could not infect humans directly. However, the fact that a highly pathogenic H5N1 avian influenza virus was transmitted directly from chickens to humans in Hong Kong, first reported in 1997, proved that avian influenza viruses could infect humans directly. There have been some inconsistencies in the literature regarding the distribution characteristics of influenza virus receptors in respiratory tract tissues of human and different animals. To accurately determine the distribution and expression characteristics of two types of influenza virus receptors in trachea and lung tissues of human, mouse, chicken and duck, we analyzed lectin immunofluorescence staining results of various tissue sections qualitatively and quantitatively. Results from qualitative analysis showed that both influenza virus receptors were expressed in lung tissues of humans, mice, chickens and ducks as well as trachea 
Table 3 Results of quantitative analysis of two influenza virus sialic acid receptors in lung tissues of humans, mice, chickens and ducks

\begin{tabular}{|c|c|c|c|c|}
\hline & Human & Mouse & Chicken & Duck \\
\hline & $\begin{array}{l}\text { Positive cells/cells } \\
\text { counted }(\%)\end{array}$ & $\begin{array}{l}\text { Positive cells/cells } \\
\text { counted }(\%)\end{array}$ & $\begin{array}{l}\text { Positive cells/cells } \\
\text { counted }(\%)\end{array}$ & $\begin{array}{l}\text { Positive cells/cells } \\
\text { counted }(\%)\end{array}$ \\
\hline MAA-I $(\alpha-2,3)$ & $379 / 8434(4.5 \%)$ & $302 / 8636(3.5 \%)$ & $707 / 16174(4.4 \%)$ & $159 / 5853(2.7 \%)$ \\
\hline MAA-II $(\alpha-2,3)$ & $344 / 7407(4.6 \%)$ & $382 / 10350(3.7 \%)$ & $468 / 12460(3.8 \%)$ & $160 / 6194(2.6 \%)$ \\
\hline $\operatorname{SNA}(\alpha-2,6)$ & $348 / 8255(4.2 \%)$ & $349 / 8525(4.1 \%)$ & $535 / 10301(5.2 \%)$ & $138 / 5352(2.6 \%)$ \\
\hline PBS control & $220 / 6973(3.2 \%)$ & $124 / 3024(4.1 \%)$ & $298 / 13306(2.2 \%)$ & $126 / 5607(2.2 \%)$ \\
\hline
\end{tabular}

tissues of mice and ducks. However, the human influenza virus receptor was expressed only in the human trachea tissue and avian influenza virus receptor was expressed only in the chicken trachea tissue (Table 1). Results of our qualitative analysis were basically consistent with some previous studies [9-11], and different from others [12,13], which may be related to the use of different reagents and detection methods. Results from the quantitative analysis demonstrated that both receptors were expressed in trachea tissues of humans and mice, as well as in lung tissues of humans, chickens and ducks; however, rates of expression were different. These results suggest that trachea tissues of humans and mice, as well as lung tissues of humans, chickens and ducks could likely be infected by human and avian influenza viruses. During the quantification process, we found that there were some false positive cells in blank control samples, which may be attributed to the amount of endogenous biotins which exist in the normal animal tissues, and could be easily mistaken for specific binding of sialic acid receptors.

When inconsistencies arise from quantitative and qualitative analyses, we believe results from the quantitative analysis should prevail. The reason causing the inconsistency between qualitative and quantitative analyses of influenza virus receptors is that the qualitative method used in this study, as well as in some other previously reported studies was based on the analysis of a small number of lectin or influenza virus stained cells observed under a microscope. Consequently, conclusions obtained by this method often had limitations. In addition, during the operational procedures of qualitative analysis, experimental results of lectin or influenza virus immunohistochemistry staining are also more vulnerable to subjective factors depending on the observer. Moreover, there is a lack of a standardized protocol for the systematic comparison of different studies. Therefore, we believe that conclusions on the distribution characteristics of the two types of influenza virus sialic acid receptors in respiratory tract tissues of different animals need to be reevaluated. A standardized protocol could be established for the quantitative analysis of lectin or influenza virus immunohistochemistry results, which will enable a more accurate description of the real distribution status of these receptors in a variety of host tissues.

It can be seen from studies of other types of receptors, that the number and density of receptors could affect their biological functions. For example, results using real-time quantitative PCR to analyze the replication difference of Helicoverpa armigera single nuclear polyhedrosis virus (HearNPV) in infected exponential and stationary HzAM1 cells showed that the amount of virus entering cells and the final progeny virions in the exponential phase cells were clearly higher than those in the infected stationary phase cells. These results suggest that the amount of virus entering cells is correlated to the number of active virus receptors on the surface of healthy cells [14]. Studies on acetylcholine receptor [15], somatostatin receptor [16], vitamin D receptor [17], IL-7 receptor [18], AMPA receptors [19] and CCR5 receptor [20] and others all indicated that the number of receptors was changed dynamically depending on the physiological status of the cell. The number of receptors affected their biological functions, and only when they reached a certain number receptors would they play their respective biological roles. Furthermore, the increased number of receptors may also be a pathogenic factor of certain diseases. Based on these findings, we predict that influenza virus receptors are probably expressed and widespread in the respiratory tract tissues of different animals. The number of expressed receptors and their local densities may vary depending on differences in localization, time and physiological environment, leading to their different susceptibilities to a variety of influenza viruses. Therefore, we suggest that scientists do not use positive or negative staining to describe the distribution status of influenza virus receptors when examining host tissues.

In conclusion, quantitative analysis is more accurate in interpreting experimental results, and an established standardized system will allow results from different laboratories to be comparable. Results from our quantitative analysis indicated that the distribution and expression status of influenza virus receptors in trachea and lung tissues of human, mouse, chicken and duck are not simply the distinction between the presence or absence of receptors, but rather the difference in the amount of expressed receptors. Here, the side-by-side comparison of two methods suggests that the use of a quantitative method to analyze immunofluorescence staining results is more reliable, aiding us to better understand the mechanism of influenza virus infection and cross-species transmissions.

The authors thank the Department of Pathology, Affiliated Hospital of Zunyi Medical College for providing paraffin-embedded human lung tissue sections, and Wang JianHua of the Laboratory of Respiratory Medicine, 
Department of Respiratory Medicine, Affiliated Hospital of Zunyi Medical College for her help during the collection of tissue samples of chickens and ducks. This work was supported by the Governor's Fund of Guizhou Province for Outstanding Individuals in Science, Technology and Education (Grant No. 2010-68).

1 Korteweg C, Gu J. Pathology, molecular biology, and pathogenesis of avian influenza A (H5N1) infection in humans. Am J Pathol, 2008, 172: 1155-1170

2 Tollis M, Di Trani L. Recent developments in avian influenza research: epidemiology and immunoprophylaxis. Vet J, 2002, 164: 202-215

3 Ge S, Wang Z. An overview of influenza A virus receptors. Crit Rev Microbiol, 2011, 37: 157-165

4 Zhang H. Tissue and host tropism of influenza viruses: Importance of quantitative analysis. Sci China Ser C-Life Sci, 2009, 52: 1101-1110

5 Wang W C, Cummings R D. The immobilized leukoagglutinin from the seeds of Maackia amurensis binds with high affinity to complex-type Asn-linked oligosaccharides containing terminal sialic acid-linked alpha-2,3 to penultimate galactose residues. J Biol Chem, 1988, 263: 4576-4585

6 Mach L, Scherf W, Ammann M, et al. Purification and partial characterization of a novel lectin from elder (Sambucus nigra L.) fruit. Biochem J, 1991, 278: 667-671

7 Zhou G Y. Histopathological Technology. Beijing: Peking University Medical Press, 2006. 1-72

8 Centers for Disease Control and Prevention (CDC). Isolation of avian influenza A (H5N1) viruses from humans-Hong Kong, May-December 1997. MMWR Morb Mortal Wkly Rep, 1997, 46: 1204-1207

9 Ning Z Y, Luo M Y, Qi W B, et al. Detection of expression of influenza virus receptors in tissues of BALB/c mice by histochemistry. Vet Res Commun, 2009, 33: 895-903
10 Ning Z Y, Liu X M, Luo M Y. Distribution of influenza virus receptors in trachea and lung of three animal detected by histochemistry. Chin J Animal Health Inspect, 2009, 4: 44-47

11 Kuchipudi S V, Nelli R, White G A, et al. Differences in influenza virus receptors in chickens and ducks: Implications for interspecies transmission. J Mol Genet Med, 2009, 3: 143-151

12 Gambaryan A, Webster R, Matrosovich M. Differences between influenza virus receptors on target cells of duck and chicken. Arch Virol, 2002, 147: 1197-1208

13 Ibricevic A, Pekosz A, Walter M J, et al. Influenza virus receptor specificity and cell tropism in mouse and human airway epithelial cells. J Virol, 2006, 80: 7469-7480

14 Dai W T, Han X, Wang H L. Analysis difference of HearNPV duplication in log phase and platform. Chin J Virol, 2007, 23: 399-406

15 Yang B, Zhou Y C, Li S T. Effects of acute denervation on ratio change of nicotinic acetylcholine receptor subtypes in the rat gastrocnemius muscle. J Pract Med, 2008, 24: 4012-4014

16 Zhang X H, Chen Z P. The application of somatostatin analogues in the patients with $\mathrm{GH}-$ secreting pituitary adenoma. Chin J Neurooncol, 2008, 6: 69-71

17 Deng Y L, Li C Y. Advance in the study of the idiopathic hypercalciuria. Int J Urol Nephrol, 2007, 27: 371-374

18 Vudattu N K, Kuhlmann-Berenzon S, Khademi M, et al. Increased numbers of IL-7 receptor molecules on CD4+CD25-CD107a+ T-cells in patients with autoimmune diseases affecting the central nervous system. PLoS ONE, 2009, 4: e6534

19 Tanaka J, Matsuzaki M, Tarusawa E, et al. Number and density of AMPA receptors in single synapses in immature cerebellum. $\mathrm{J}$ Neurosci, 2005, 25: 799-807

20 Desmetz C, Lin Y L, Mettling C, et al. The strength of the chemotactic response to a CCR5 binding chemokine is determined by the level of cell surface CCR5 density. Immunology, 2006, 119: 551-561

Open Access This article is distributed under the terms of the Creative Commons Attribution License which permits any use, distribution, and reproduction in any medium, provided the original author(s) and source are credited. 